TITLE:

\title{
Characterization of Plasma Process-Induced Latent Defects in Surface and Interface Layer of Si Substrate
}

\author{
$\operatorname{AUTHOR(S):~}$
}

Nakakubo, Yoshinori; Eriguchi, Koji; Ono, Kouichi

\section{CITATION:}

Nakakubo, Yoshinori ...[et al]. Characterization of Plasma Process-Induced Latent Defects in Surface and Interface Layer of Si Substrate. ECS Journal of Solid State Science and Technology 2015, 4(6): N5077-N5083

ISSUE DATE:

2015-02-14

URL:

http://hdl.handle.net/2433/210585

\section{RIGHT:}

(c) The Author(s) 2015. Published by ECS. This is an open access article distributed under the terms of the Creative Commons Attribution Non-Commercial No Derivatives 4.0 License (CC BY-NC-ND,

http://creativecommons.org/licenses/by-nc-nd/4.0/), which permits non-commercial reuse, distribution, and reproduction in any medium, provided the original work is not changed in any way and is properly cited. For permission for commercial reuse, please email: oa@electrochem.org. 


\title{
Characterization of Plasma Process-Induced Latent Defects in Surface and Interface Layer of Si Substrate
}

\author{
Yoshinori Nakakubo, ${ }^{\text {a,b }}$ Koji Eriguchi, ${ }^{a, z}$ and Kouichi Ono ${ }^{a}$ \\ ${ }^{a}$ Graduate School of Engineering, Kyoto University, Kyoto 615-8540, Japan \\ ${ }^{b}$ Semiconductor \& Storage Products Company, Toshiba Corporation, Mie 512-8550, Japan
}

\begin{abstract}
Characterization of plasma-induced Si substrate damage is demonstrated using an electrical capacitance-voltage $(C-V)$ technique customized for the nano-scale analysis. Low resistive $\mathrm{Si}$ wafers are exposed to an inductively coupled plasma (ICP) or a capacitively coupled plasma (CCP). We focus on the effects of plasma parameters and wet-etching processes on plasma-induced physical damage (PPD) analyses. The optical thicknesses of surface and interfacial layers $\left(d_{\mathrm{SL}}\right.$ and $\left.d_{\mathrm{IL}}\right)$ were characterized using spectroscopic ellipsometry (SE) and compared with the electrical oxide thicknesses (EOT) obtained by the $C-V$ technique. In the case of asdamaged samples, the optical thickness $d_{\mathrm{SL}}$ by SE is found to be smaller than the EOT by the $C-V$ technique, while the sum of $d_{\mathrm{SL}}$ and $d_{\mathrm{IL}}$ was approximately equal to the EOT. A diluted hydrofluoric acid (DHF) wet-etch step is employed to address depth profile of defect density in damaged samples. We identify the latent defect density, $d_{\mathrm{SL}}$, and $d_{\mathrm{IL}}$ after the DHF wet-etch, which are indispensible for practical device performance designs. It is found that, although the average energy of incident ions $\left(\bar{E}_{\text {ion }}\right)$ is larger for the case of CCP, the latent defect density of CCP-damaged samples is smaller than that of ICP even after the wet-etching. This finding is in sharp contrast to previous pictures - the larger $\bar{E}_{\text {ion }}$ leads to the thicker damaged layer and the larger latent defect density. We propose a model for these conflicting results, where the profiles of defect density and the sensitivities of each analysis technique are taken into account. The present work highlights the importance of the nano-scale damage characterization using the $C-V$ technique, allowing to understand the influence of latent defects and to enable better design of future electronic devices.

(C) The Author(s) 2015. Published by ECS. This is an open access article distributed under the terms of the Creative Commons Attribution Non-Commercial No Derivatives 4.0 License (CC BY-NC-ND, http://creativecommons.org/licenses/by-nc-nd/4.0/), which permits non-commercial reuse, distribution, and reproduction in any medium, provided the original work is not changed in any way and is properly cited. For permission for commercial reuse, please email: oa@electrochem.org. [DOI: 10.1149/2.0121506jss] All rights reserved.
\end{abstract}

Manuscript submitted January 29, 2015; revised manuscript received March 30, 2015. Published April 8, 2015. This paper is part of the JSS Focus Issue on Atomic Layer Etching and Cleaning.

Plasma processing plays an important role in manufacturing present-day microelectronics. Over the last two decades, plasma process-induced damage (PID) has been one of the crucial problems in fabricating metal-oxide-semiconductor field-effect transistors (MOSFETs) $)^{1-3}$ in LSI (Large Scale Integration) circuits. PID consists of four major mechanisms. ${ }^{2}$ The first mechanism is damage induced by conduction current from plasma flowing into MOSFETs, resulting in degradation of the performance and increase in the parameter variability owing to plasma-induced electrical stress. ${ }^{1,4,5}$ This electrical interaction has been discussed and is called plasma-induced charging damage. ${ }^{2}$ The second mechanism is damage induced by incident photons on material surface. Photons with high energy can interact with materials such as photoresist masks and low-k dielectrics, leading to bond breaking in the materials exposed to plasma, or in some cases, create an interface state between $\mathrm{SiO}_{2}$ and $\mathrm{Si}$ substrate. ${ }^{6-8}$ The third mechanism is surface and bulk chemical reaction with fast diffusion reactions causing material modification such as surface defects and roughness. The fourth mechanism is damage induced by high-energy ion bombardment on Si substrates or other materials. ${ }^{9-14}$ In this paper, we focus on the fourth mechanism in Si substrates, which we denote as "plasma-induced physical damage (PPD)" for simplicity. PPD usually creates "defects", ${ }^{10,14,15}$ such as $\mathrm{Si}$-Si broken or strained bonds, displaced $\mathrm{Si}$ atoms, vacancies, and interstitial atoms in crystalline Si substrates. The PPD on Si substrate surfaces has been extensively studied recently, because the mechanism of PPD is naturally governed by basic plasma parameters such as sheath structure, plasma density, and so on. For example, a Si recess ${ }^{11,12,16}$ formed on the source/drain extension region in a MOSFET is considered to have a negative impact on device performance such as threshold voltage shift. ${ }^{16}$ Moreover, the latent defects remaining after a wet-etch step following plasma etching degrades drain saturation current $\left(I_{\mathrm{on}}\right)$, which means device operation slow down. ${ }^{15,17}$ This damage creation mechanism was clarified using the modified range theory. ${ }^{18}$ Eriguchi et al. recently modeled ${ }^{18}$

${ }^{\text {zE} E-m a i l: ~ e r i g u c h i @ k u a e r o . k y o t o-u . a c . j p ~}$ the dependence of the surface damaged-layer thickness on the incident average ion energy $\bar{E}_{\text {ion }}$ and rf-bias frequency applied to a wafer stage. The thickness of the damaged layer showed a power-law dependence on the average ion energy $\bar{E}_{\text {ion }} \cdot{ }^{18-20}$ Since the thickness of the damaged layer may be equivalent to the Si recess depth $\left(d_{\mathrm{R}}\right), d_{\mathrm{R}}$ is considered to be governed significantly by $\bar{E}_{\text {ion }}$. Thus, controlling the $\bar{E}_{\text {ion }}$ is the principal approach for suppressing the PPD. For example, a low ion energy process such as atomic layer etching (ALEt) has been extensively studied for this purpose. ${ }^{21}$

Various damage characterization techniques have been introduced so far. Among them, spectroscopic ellipsometry (SE) is widely used as in-line monitoring for PPD measurement. One usually observes an increase in the thicknesses of surface (SL) and/or interfacial (IL) layers in sub-nm scale. In the analysis, the sensitivity relies on the observed changes in dielectric constants and other optical parameters such as an extinction coefficient. Using SE, several reports discussed depth profiles of dielectric constant change. ${ }^{22-26}$ For example, $\mathrm{H}$-plasma damaged samples were found to have relatively thick IL $\sim 10 \mathrm{~nm},{ }^{14,27}$ which was difficult to remove by wet-etching. ${ }^{14}$ These findings related to SL and IL are of great importance to device designs under process constraints, because the final topological structure of MOSFETs with a Si recess defines the performance. Regarding defect sites created by PPD, electrical measurements have been widely performed. ${ }^{28-33}$ Among them, a $C-V$ technique has been primarily proposed $^{34}$ to probe the density $\left(n_{\mathrm{dam}}\right)$ in damaged structures. Historically, there have been many studies conducted so far using $C-V$ techniques in ion-implantation or other processes ${ }^{35-37}$ for the purpose of quantifying dopant density. Preliminary exploring of the $C-V$ technique to $\mathrm{PPD}^{34,38}$ led to the conclusion that, under typical plasma etching processes, $n_{\mathrm{dam}}$ ranges from $10^{18}$ to $10^{19} \mathrm{~cm}^{-3}$. In addition to $n_{\text {dam }}$ of as-damaged samples, addressing the residual $n_{\text {dam }}$ after a wetetching step is indispensible to design drain current of a MOSFET, because $n_{\text {dam }}$ directly affects the resistance change in the source/drain extension region. However there have been only few discussions regarding the residual $n_{\text {dam }}$ after a wet-etching. A methodology to evaluate quantitatively the $n_{\text {dam }}$ profile is requisite to design a device of the 
final structure in ultimately scaled regime as well as to understand the damage creation for the low ion energy case such as ALEt process. In this study, we demonstrate a comprehensive evaluation of the PPD profile using $\mathrm{SE}$ and a customized $1 / C^{2}-V$ method capable to quantify $n_{\text {dam }}$ in the nano-scale regime. After briefly reviewing a PPD model, surface structures of plasma-exposed $\mathrm{Si}$ substrates are investigated in detail by focusing on the profiles of $n_{\mathrm{dam}}$ by using wet-etching. By comparing the obtained data for inductively coupled plasma (ICP) or capacitively coupled plasma (CCP) exposures, we discuss the impacts of the ion flux on the damage characterization. Key issues in the nano-scale damage characterization are presented.

\section{PPD Model-PPD Range Theory}

Si substrate damage in a MOSFET was modeled by means of the so-called range theory, ${ }^{39}$ as follows. The distribution range of injected ions can be determined from the stopping power. ${ }^{39}$ The total distance that an ion travels before coming to rest is simply called its range $R$, and the projection of this distance along the axis of incidence, the projected range $R_{\mathrm{p}}$. In typical plasma processes, the energy of ions is much lower than that in ion implantation processes. ${ }^{40,41}$ Thus, one can focus solely on the nuclear stopping process. ${ }^{39,42}$ From the modified range theory for PPD,${ }^{18}$ the spatial distribution of impinging ions in the $\mathrm{Si}$ substrate is expressed by a characteristic profile, where both $R_{\mathrm{p}}$ and the straggling $\sigma_{\mathrm{p}}{ }^{2}$ are defined. ${ }^{19,40,42,43} R_{\mathrm{p}}$ is expressed as

$$
R_{\mathrm{p}}=A_{\mathrm{PPD}} \cdot\left(\bar{E}_{\mathrm{ion}}\right)^{\alpha}
$$

where $A_{\mathrm{PPD}}$ and $\alpha$ are material-specific constants. The recess depth $d_{\mathrm{R}}$ is also a function of $\bar{E}_{\text {ion }}$ and wet-etch time $t_{\text {wet }} \cdot{ }^{44}$ For a given $t_{\text {wet }}, d_{\mathrm{R}}$ is generally expressed by

$$
d_{\mathrm{R}}=B_{\mathrm{PPD}} \cdot\left(\bar{E}_{\mathrm{ion}}\right)^{\beta}
$$

where $B_{\mathrm{PPD}}$ and $\beta$ are material- and process-specific constants. The spatial distribution of injected ions around the depth $R_{\mathrm{p}}$ is usually approximated as a Gaussian distribution with a standard deviation $\sigma_{\mathrm{p}}$.

In the modern manufacturing processes, a subsequent wet-etch is performed after a plasma step in order to remove contaminants from the plasma-exposed surface. ${ }^{45}$ Sometimes residual defects remain after the wet-etch step, however, a longer wet etch time leads to a larger $d_{\mathrm{R}}$. Since the areal density of residual defects $N_{\mathrm{dam}}$ is determined from the original profile of defects in an as-damaged sample $\left(n_{\mathrm{dam}}^{0}\right)$ and the wet-etch depth $\left(=d_{\mathrm{R}}\right)$, the relationship between $N_{\mathrm{dam}}$ and $d_{\mathrm{R}}$ is expressed as

$$
N_{\mathrm{dam}}=\int_{d_{R}}^{\infty} n_{\mathrm{dam}}^{0}(x) d x .
$$

Note that $N_{\mathrm{dam}}$ decreases with an increase in $d_{\mathrm{R}}$ under a given $n_{\mathrm{dam}}^{0}$.

The conclusions derived from the discussion above have been verified from the measurement of damaged thickness by $\mathrm{SE}^{24}$ or damage profiles of incident species by SIMS (Secondary Ion Mass Spectroscopy). ${ }^{18}$ As shown below, we demonstrate a comprehensive evaluation of the defect density for as-damaged and wet-etched samples.

\section{Experimental}

Plasma reactors and sample treatment.- 4-inch n-type (100) Si wafers with resistivity of $0.02 \Omega \mathrm{cm}$ were exposed to Ar-based ICP and CCP discharges as shown in Figs. 1 for 30 s. The unexposed sample is denoted as "Ref" in this study. The processing time of $30 \mathrm{~s}$ was chosen as a saturation value in terms of the damaged-layer formation process according to the previous results. ${ }^{46,47}$ Table I shows process conditions employed in this study. RF biasing at $13.56 \mathrm{MHz}$ was applied to the wafer stage $120 \mathrm{~mm}$ in diameter with powers ranging from 50 to $200 \mathrm{~W}$ in the ICP or with a power of $10 \mathrm{~W}$ in the CCP, respectively. The ICP source power was $100 \mathrm{~W}$. Plasma diagnostics using a Langmuir probe and an oscilloscope determined the plasma density and the average self dc bias $\left(V_{\mathrm{dc}}<0\right)$. Ion fluxes $\left(\Gamma_{\mathrm{ion}}\right)$ to the Si substrate were estimated from saturation ion current monitored

$\begin{aligned} & \text { Table I. Process conditions and plasma parameters in this study. } \\
& \text { (Ar gas, 2.7 Pa, 30 s, ICP source power: } 100 \mathrm{~W}) .\end{aligned}$
\begin{tabular}{ccccc} 
Source & $\begin{array}{c}\text { Bias power } \\
\text { (W) }\end{array}$ & $\begin{array}{c}\text { Electron } \\
V_{\mathrm{dc}}(\mathrm{V})\end{array}$ & $\begin{array}{c}\text { Ion flux } \\
\left(\mathrm{cm}^{-2} \mathrm{~s}^{-1}\right)\end{array}$ \\
\hline ICP & 25 & -34 & & \\
& 50 & -70 & & \\
& 75 & -90 & $1.0 \times 10^{11}$ & $3.1 \times 10^{14}$ \\
& 100 & -130 & & \\
$\mathrm{CCP}$ & 200 & -240 & & \\
& 10 & -260 & $4.0 \times 10^{9}$ & $1.2 \times 10^{13}$
\end{tabular}

by an oscilloscope as listed in Table I. The average ion energy $\bar{E}_{\text {ion }}$ is defined as $q\left(V_{\mathrm{p}}-V_{\mathrm{dc}}\right)$ ( $q$ is the elementary charge) from the estimated plasma potential.

In order to study the effects of wet clean, damaged samples were immersed in $\cong 0.1 \%$ diluted hydrofluoric acid (DHF) for $60 \mathrm{~s}$, followed by $\mathrm{SE}$ and $C-V$ measurements (as mentioned below) immediately, and then immersed again in DHF for additional 300 s. Finally SE and

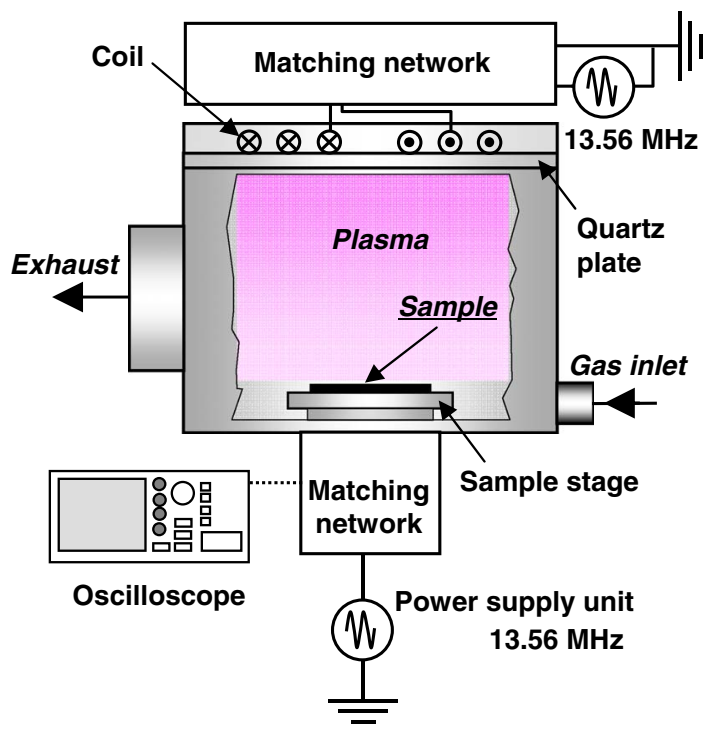

(a)

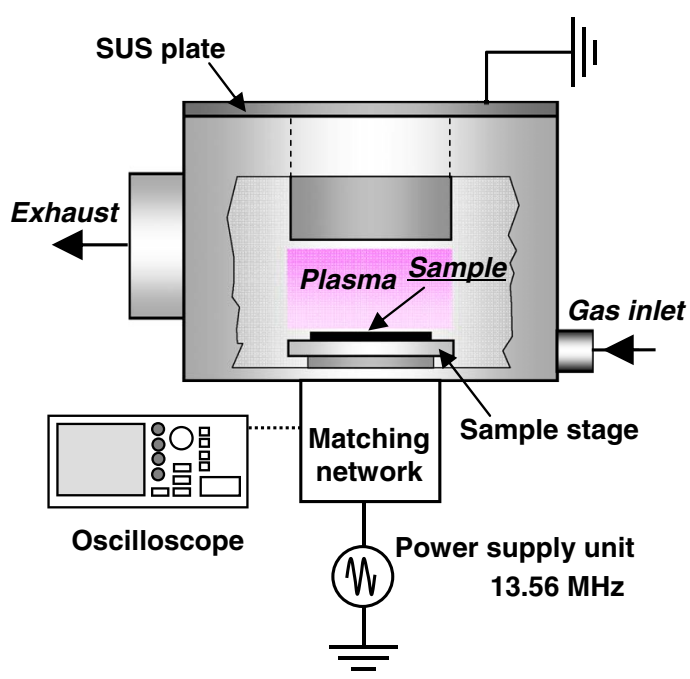

(b)

Figure 1. (a) Inductively coupled plasma system used in this study. The source power applied through a 3-turn coil was $100 \mathrm{~W}$. Space gap between the quartz plate and the stage is $100 \mathrm{~mm}$. (b) Capacitively coupled plasma system used in this study. Space gap between the top electrode and the stage is $47 \mathrm{~mm}$. 


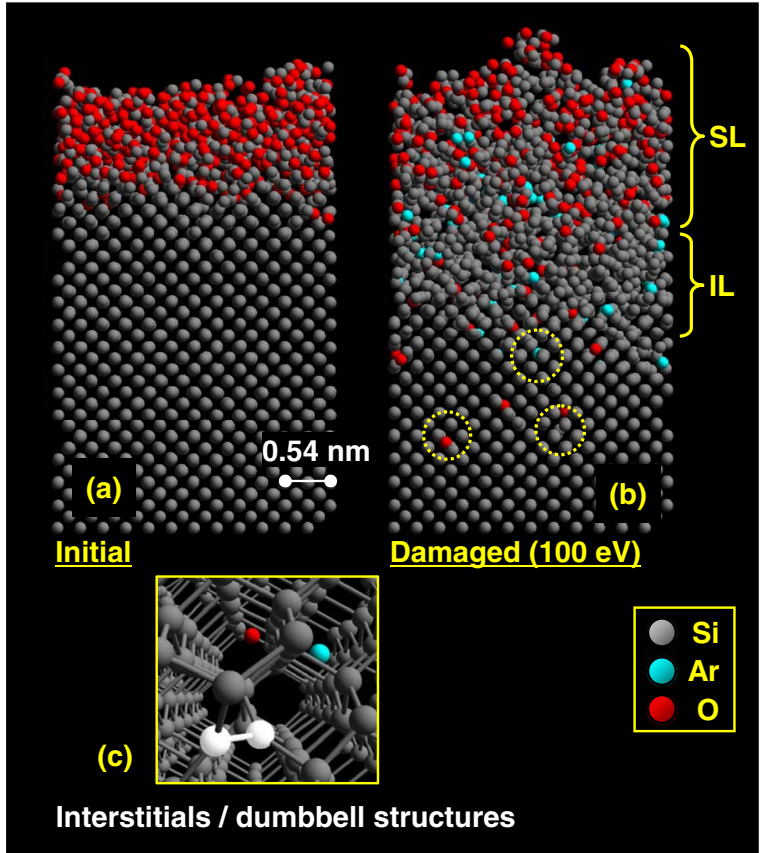

Figure 2. MD simulation results for (a) a starting $\mathrm{SiO}_{2} / \mathrm{Si}$ structure and (b) a damaged structure after Ar-impact $\left(E_{\text {ion }}=100 \mathrm{eV}\right)$. In addition to the damaged layer near the reaction surface, local defects are observable beneath the IL region. (c) Typical defect structures, i.e., interstitials and dumbbell structures.

$C-V$ measurements were performed to assign the final thickness and residual defect densities for all samples, respectively.

Optical characterizations. - To clarify the structure of damaged layers and the thickness, spectroscopic ellipsometry (SE) was used. In this study, measurements were carried out in the photon energy range of $1.60-5.50 \mathrm{eV}$ at $0.05 \mathrm{eV}$ intervals. As analyzed by highresolution TEM (HR-TEM), Rutherford backscattering spectroscopy (HR-RBS), ${ }^{24}$ and molecular dynamic (MD) simulations ${ }^{48}$ the damaged layer consists of two regions, i.e., the surface and interfacial layers (abbreviated SL and IL, respectively). Figure 2 shows one of typical damaged structures including local defects, predicted by MD simulation. The MD code used here was originally developed by Ohta and Hamaguchi. ${ }^{49}$ Further details of the MD procedure are described elsewhere. ${ }^{49,50}$ This simulation was performed for Ar-atom impacts using an $\mathrm{SiO}_{2} / \mathrm{Si}$ substrate as a staring structure (Fig. 2a). We employed the potential model presented by Wilson et al. ${ }^{51}$ for the $\mathrm{Si}-\mathrm{Ar}$ system. The periodic boundary condition was employed along the horizontal direction. An atom regarded as an ion was injected from randomly selected horizontal locations above the surface at normal incidence. One thousand ions impinged into this structure. As indicated in Fig. 2b, the $\mathrm{SL}$ is primarily composed of $\mathrm{SiO}_{2}$, as a result of oxidation of heavily damaged regions by exposure to air and the presence of knock-on oxygen from the native oxide layer. The IL is a partially oxidized or disordered Si layer, i.e., a mixed layer consisting of crystalline $\mathrm{Si}$ and $\mathrm{SiO}_{2}$ phases. Based on the previous report, ${ }^{26}$ in $\mathrm{SE}$ analysis, an optical model assuming four layers (air / SL / IL / Si substrate) was employed. We applied effective medium approximation (EMA) to define the IL. To obtain the surface (SL: $d_{\mathrm{SL}}$ ) and interfacial [IL $\left(\mathrm{SiO}_{2}+\right.$ crystalline-Si): $d_{\mathrm{IL}}$ ] layer thicknesses and the composition, the spectrum was fitted with the optical model by regression analysis to minimize the unbiased estimator. The thickness of the damaged layers defined as $d_{\mathrm{SL}}+d_{\mathrm{IL}}$ is the principal outcome of the SE measurement. Note that localized structures as indicated in Fig. 2c cannot be detected by SE. These defect structures can be identified by the $C-V$ method.

Electrical characterization technique.- We performed an electrical characterization using a mercury $(\mathrm{Hg})$ probe system. An LCR- meter (HP-4284) was used to measure $C-V$ characteristics of damaged structures $\left(\mathrm{Hg} / \mathrm{SiO}_{2} / \mathrm{Si}\right)$. The frequency of voltage modulation superimposed on the DC bias was $100 \mathrm{kHz}$. The electrical oxide thickness $\left(\right.$ EOT) ${ }^{45}$ was estimated from the maximum capacitance in accumulation. ${ }^{37}$ We measured the capacitance in inversion for determining the defect density $\left(n_{\mathrm{dam}}\right)$ of damaged samples. In accumulation, the measured differential capacitance corresponds to that of $\mathrm{SiO}_{2}$ layer ( $\left.\sim \mathrm{SL}, C_{\mathrm{SL}}\right)$ because the wafer resistivity is low enough $(0.02 \Omega \mathrm{cm})$. In inversion, the measured capacitance corresponds to the sum of $C_{\mathrm{SL}}$ and the inversion layer capacitance. In this configuration, the slope of $1 / C^{2}-V$ is a function of the density of doping $\left(N_{\mathrm{D}}\right)$ of the bulk substrate (in the present case, $N_{\mathrm{D}} \sim 10^{18} \mathrm{~cm}^{-3}$ ). The low resistivity of our wafers allows to measure the differential capacitance in the inversion layer close to the surface (approximately $5 \mathrm{~nm}$ thick). When a Si substrate is exposed to a plasma, defects with various structures are formed in the SL and IL. As shown in Fig. 2c, after thorough inspection of the local defect structures in the MD simulation snapshots, typical structures commonly observed were interstitial atoms ${ }^{52,53}$ and a dumbbell type $^{38,54,55}$ Note that the surface amorphized ${ }^{56}$ and interlayer regions in Fig. $2 b$ do correspond respectively to the SL and IL measured by SE. Since the local defects observable in MD simulation are stable structures that act as traps for carriers, ${ }^{52-55}$ the change in the inversion capacitance corresponds to the change in "effective" doping concentration. By introducing the density of defects $n_{\mathrm{dam}}$, one can describe the differential capacitance as

$$
\frac{d}{d V_{\mathrm{b}}}\left(\frac{1}{{C_{\mathrm{m}}}^{2}}\right)=-\frac{2}{q \varepsilon_{0} \varepsilon_{\mathrm{Si}}\left(N_{\mathrm{D}}+n_{\mathrm{dam}}\right)}
$$

where $C_{\mathrm{m}}$ is a measured capacitance, $\varepsilon_{\mathrm{Si}}$ is the relative dielectric constant of substrate, $\varepsilon_{0}$ is the permittivity in vacuum, and $V_{\mathrm{b}}$ is applied bias voltage. The slope of $1 / C^{2}-V$ defines $N_{\mathrm{D}}+n_{\mathrm{dam}}$. Damage causes the slope to decrease in the $V_{\mathrm{b}}$ range from -1.0 to $1.0 \mathrm{~V}$, indicating the presence of defect sites $\left(N_{\mathrm{D}}+n_{\mathrm{dam}}>N_{\mathrm{D}}\right)$. For typical plasma conditions, we estimate $n_{\text {dam }}$ to be in the range $\sim 10^{18}-10^{19} \mathrm{~cm}^{-3}$, consistent to other evaluation techniques. ${ }^{10,57}$ This is consistent with defect density found by MD simulation. Therefore, $n_{\text {dam }}$ measured by the $1 / C^{2}-V$ technique corresponds to those local defect structures beneath the IL region.

\section{Results and Discussion}

Thicknesses of damaged structures. - Figure 3 a shows $C-V$ curves of typical as-damaged samples including the Ref. For the damaged sample, the value of the capacitances in the accumulation region $\left(V_{\mathrm{b}}>\right.$ $0)$ is significantly increased. EOT is thus determined, and the results are compared with $\left(d_{\mathrm{SL}}+d_{\mathrm{IL}}\right)$ and $d_{\mathrm{SL}}$ only in Fig. $3 \mathrm{~b}$. Two key features are confirmed: $1 d_{\mathrm{SL}}$ is smaller than the EOT for all damaged samples. $2\left(d_{\mathrm{SL}}+d_{\mathrm{IL}}\right)$ is approximately equivalent to the EOT. This suggests that, in the $C-V$ measurements, $\left(d_{\mathrm{SL}}+d_{\mathrm{IL}}\right)$ is identified as a surface oxide layer having the capacitance $C_{\mathrm{ox}}$ usually extracted from MOS structures. ${ }^{37}$ Therefore, as discussed in the following, the "effective" doping concentration derived from $1 / C^{2}-V$ measurement is related to $N_{\mathrm{D}}+n_{\mathrm{dam}}$ beneath the IL by SE. This methodology enables us to quantify $n_{\text {dam }}$ in the Si substrate created by PPD.

Figure 4 shows an example for the thickness determined by SE for various samples exposed to plasmas before and after DHF wetetching. One can see decrease in $\left(d_{\mathrm{SL}}+d_{\mathrm{IL}}\right)$ after the wet-etching. Figures 5 compare the dependence of $d_{\mathrm{SL}}$ and $d_{\mathrm{IL}}$ on the average ion energy $\bar{E}_{\text {ion }}$ in the case of various ICP exposures. As seen in Fig. 5a, with an increase in $\bar{E}_{\text {ion }}, d_{\mathrm{IL}}$ monotonically increases, whereas $d_{\mathrm{SL}}$ exhibits no clear dependence on $\bar{E}_{\text {ion. }}$. This is owing to the fact that a surface damaged layer is reconstructed by air exposure (oxidation) or surface sputtering. Thus, while $d_{\mathrm{SL}}$ is independent of it, $d_{\mathrm{IL}}$ should be used as a measure of PPD. Moreover, DHF wet-etch changes $d_{\mathrm{SL}}$ and $d_{\mathrm{IL}}$ in the course of time as shown Figs. $5 \mathrm{~b}$ and $5 \mathrm{c} . d_{\mathrm{IL}}$ is significantly decreased by the 300 -s-DHF wet-etch in the lowest $\bar{E}_{\text {ion }}$ case, implying the removal of the damaged layer. 

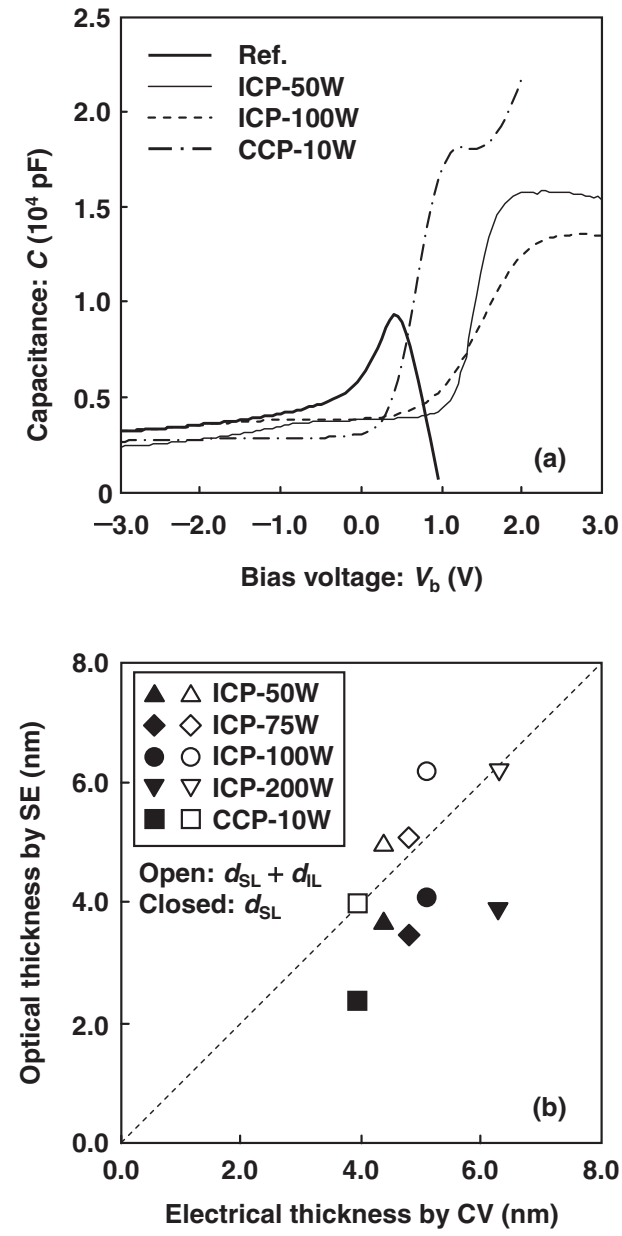

Figure 3. (a) Typical $C-V$ curves of plasma-damaged structures. The $C-V$ curve of Ref is also shown. (b) Relationship between the optical thickness by $\mathrm{SE}$ and the electrical thickness by $C-V$ for various damaged structures.

Characterization of defect density by $1 / C^{2}-V$ technique.- As discussed above, $d_{\mathrm{IL}}$ rather than $d_{\mathrm{SL}}$ should be used as a measure of PPD in SE analysis. However, no quantification of defect density can be realized only by SE. Figures 6 displays various $1 / C^{2}-V$ curves obtained before and after DHF treatments. One can see a "plateau"-gentler slope region-in damaged samples clearly. Even after the DHF treatments, the plateau remains in most cases, implying the presence of defects.

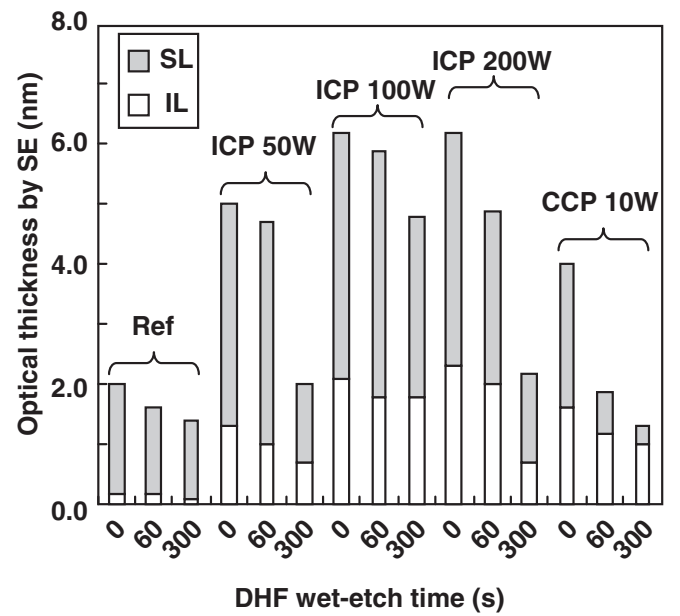

Figure 4. Example for the trend in optical thicknesses, $d_{\mathrm{SL}}$ and $d_{\mathrm{IL}}$ by DHF wet-etching for various damaged structures.
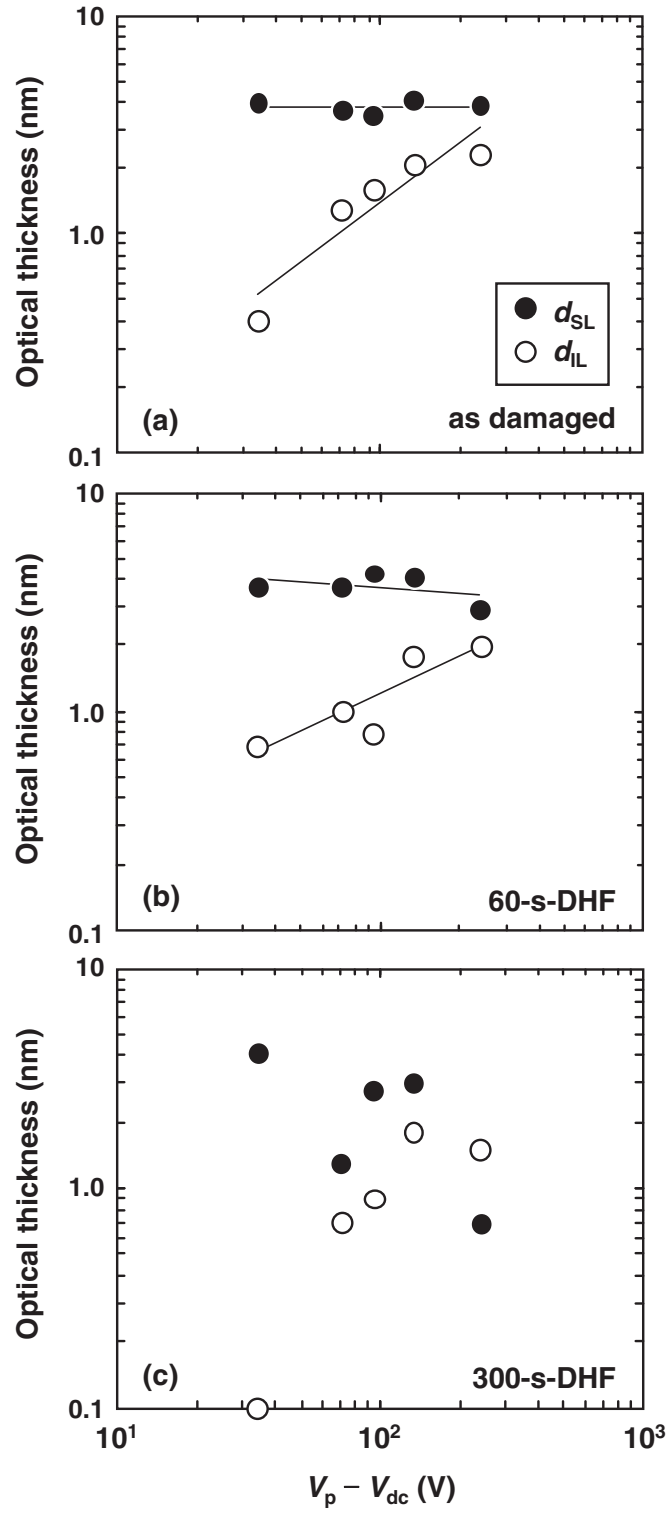

Figure 5. Dependence of $d_{\mathrm{SL}}$ and $d_{\mathrm{IL}}$ on the average incident ion energy in various DHF steps. (a) Before DHF treatment. (b) After 60-s-DHF. (c) After 300-s-DHF.

Figure 7 illustrates the mechanism explaining the presence of the plateau in the $1 / C^{2}-V$. When $V_{\mathrm{b}}$ is swept in inversion, a depletion layer is formed and extends toward deeper region of the Si substrate in accordance with $V_{\mathrm{b}}$. The intersection between the Fermi level and the defects determines the depth of defects that contribute the differential capacitance measured $\left(C_{\mathrm{m}}\right)$ at the $V_{\mathrm{b}}$. In the extent configuration such as strong inversion as shown in (C), only the dopants $\left(N_{\mathrm{D}}\right)$ can contribute to $C_{\mathrm{m}}$ because no more defects may be present in the deep substrate region. Therefore $1 / C_{\mathrm{m}}$ becomes close to $\left(1 / C_{\mathrm{ox}}\right.$ $+1 / C_{\text {inv }}$ ) where $C_{\text {inv }}$ is defined by $N_{\mathrm{D}}$. This configuration corresponds to the $1 / C^{2}-V$ curves in the inversion region. In other words, the slope becomes close to that of Ref in the strong inversion. In the intermediate $V_{\mathrm{b}}$ range as shown in (B), both dopants and defects contribute to $1 / C_{\mathrm{m}}$, i.e., the slope becomes gentler than that of Ref as Eq. 4 suggested. Moreover, the range of $V_{\mathrm{b}}$ in the plateau becomes narrower as DHF time increases, indicating a decrease in $n_{\text {dam }}$ close to the IL by the wet-etch, i.e., removal of the damaged layer and oxidation of a part of the remaining damaged layer. Figure 8 shows typical trends in $n_{\mathrm{dam}}$ for damaged samples with DHF treatments and Figure $9, n_{\mathrm{dam}}$ as a function of $\bar{E}_{\mathrm{ion}}$ for ICP damaged samples 

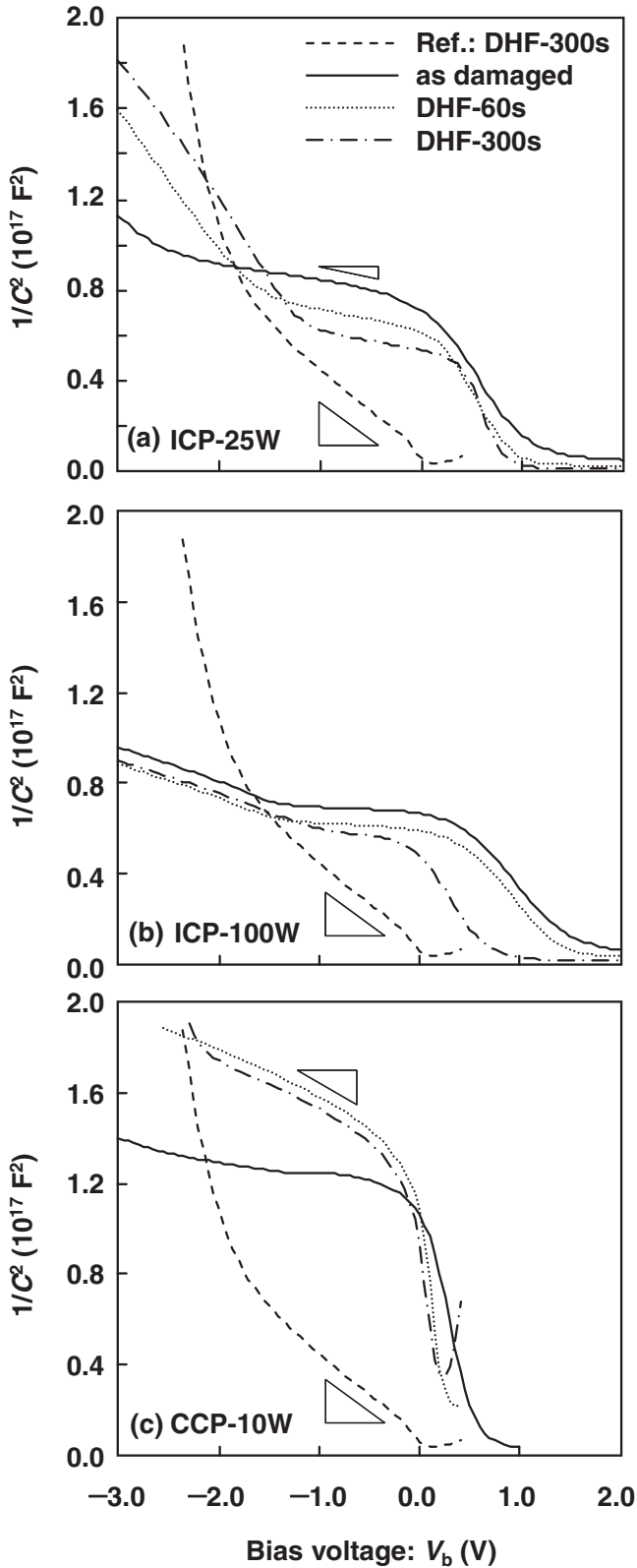

Figure 6. Typical $1 / C^{2}-V$ curves of plasma-damaged structures in various DHF steps. The $1 / C^{2}-V$ curves of Ref are also shown. (a) 25-W-ICP exposure, (b) 100-W-ICP exposure, and (c) 10-W-CCP exposure.

before and after DHF treatments. The $n_{\mathrm{dam}}$ is estimated from the slope of the plateau. Note that the $n_{\text {dam }}$ estimated by the $1 / C^{2}-V$ measurement is the volume density of residual defects in the damaged layer. The areal density $N_{\text {dam }}$ in Eq. 3 is obtained by integration of the $n_{\mathrm{dam}}^{0}$. From Figs. $9 \mathrm{a}$ and $9 \mathrm{~b}$, the $\bar{E}_{\text {ion }}$ dependence of $n_{\mathrm{dam}}$ is expressed as

$$
n_{\text {dam }}=C \cdot\left(\bar{E}_{\text {ion }}\right)^{\gamma}
$$

where $C$ and $\gamma$ are material-specific constants. In these cases, $\gamma$ is determined as 1.3 and 1.2 for the cases of Fig. $9 a$ and $9 b$, respectively.

From the presence of plateaus in the $1 / C^{2}-V$ curves in Figs. 6, even after 300-s-DHF wet-etch, it is confirmed that the residual defects are still present in the Si substrate. However, no clear $\bar{E}_{\text {ion }}$ dependence of $n_{\text {dam }}$ is seen for Fig. 9c. This feature may be attributed to the fact that residual defects are small in number (close to the dopant concentration), i.e., below the detection limit. These results imply that most of the damaged region has been oxidized and is stripped
(A) Thermal equilibrium

$$
\sim N_{\mathrm{D}} \quad \sim\left(N_{\mathrm{D}}+n_{\mathrm{dam}}\right) \quad \sim N_{\mathrm{D}}
$$

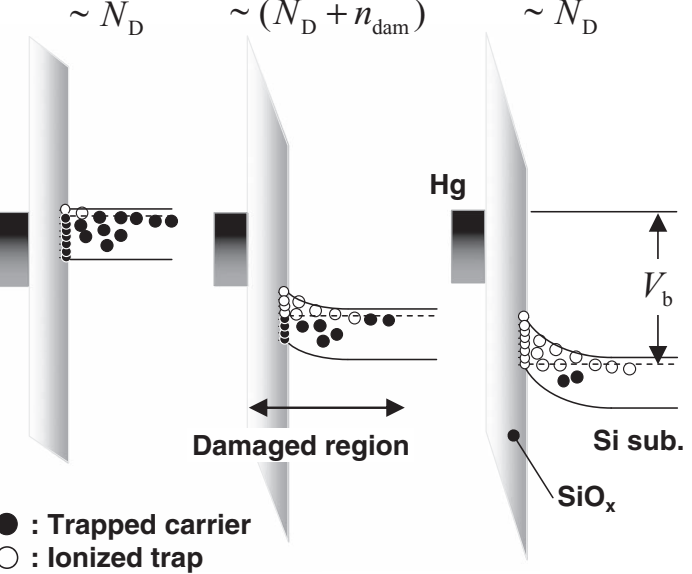

: Trapped carrier

$\bigcirc$ : lonized trap

(B) Intermediate (C) Strong inversion

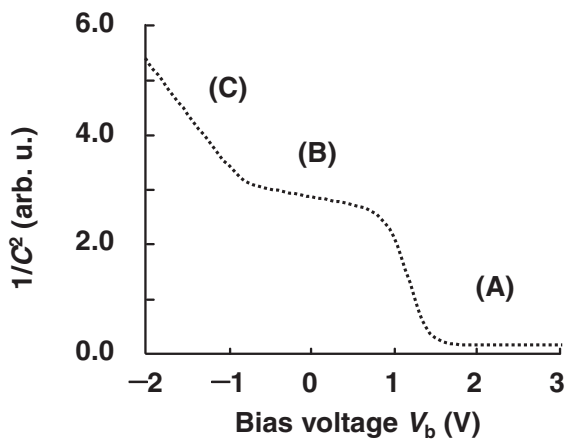

Figure 7. Energy band diagrams of a $\mathrm{Hg} / \mathrm{SiO}_{2} / \mathrm{Si}$ structure (A) in thermal equilibrium, (C) in inversion, and (B) in intermediate configuration, compared with a typical $1 / C^{2}-V$ curve.

off by 300-s-DHF wet-etch. (See the similar trend of $d_{\mathrm{IL}}$ in Figure 5.) Based on the $1 / C^{2}-V$ methodology, one can quantify the evolution of $n_{\text {dam }}$ with various processing steps such as wet-etch stripping.

Impacts of defect profile on PPD characterization methodology.Based on the PPD range theory, the defects distributed in the Si substrate have a profile defined primarily by the energy and the flux

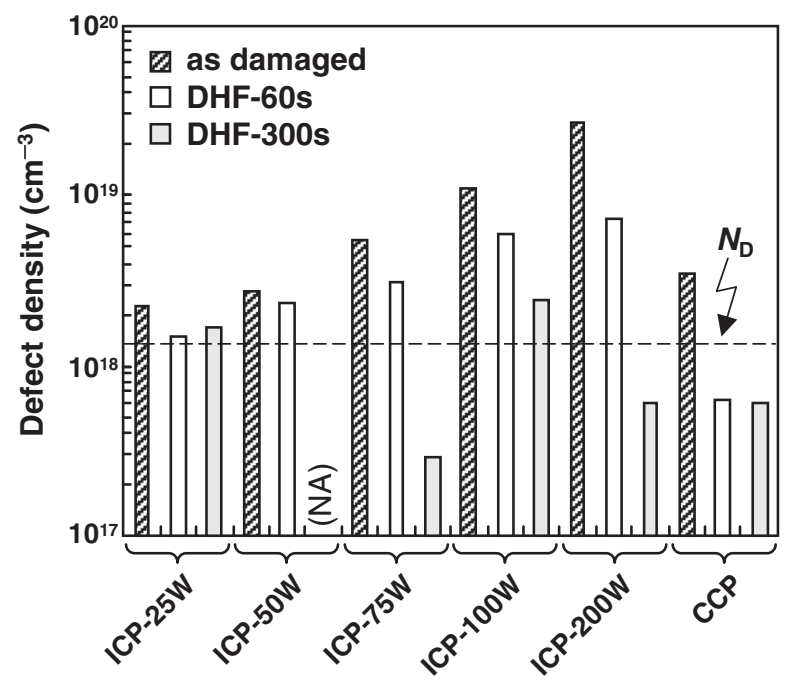

Figure 8. Example for the trend in defect densities for damaged structures in various DHF steps. 


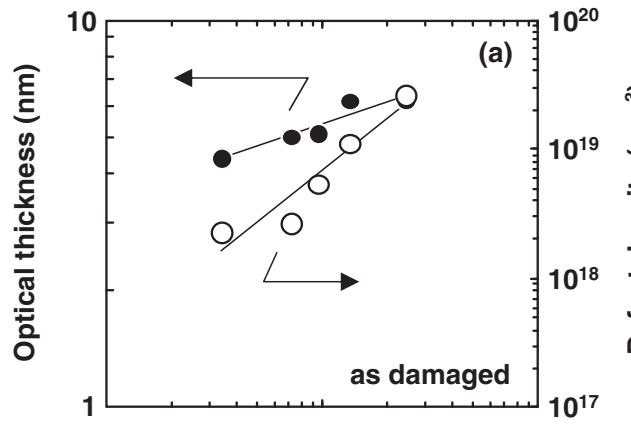

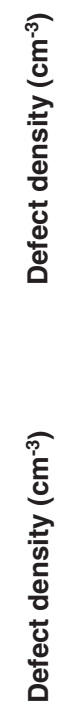

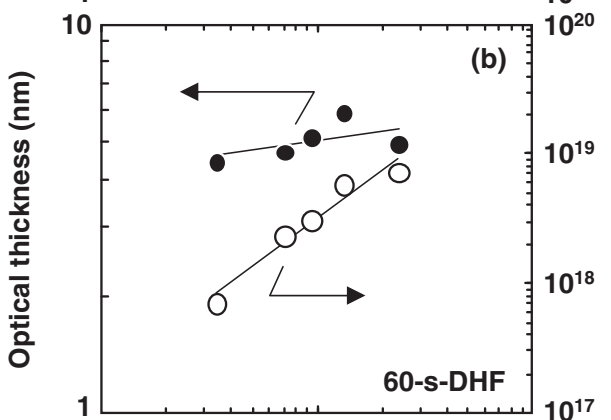

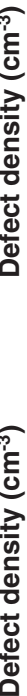

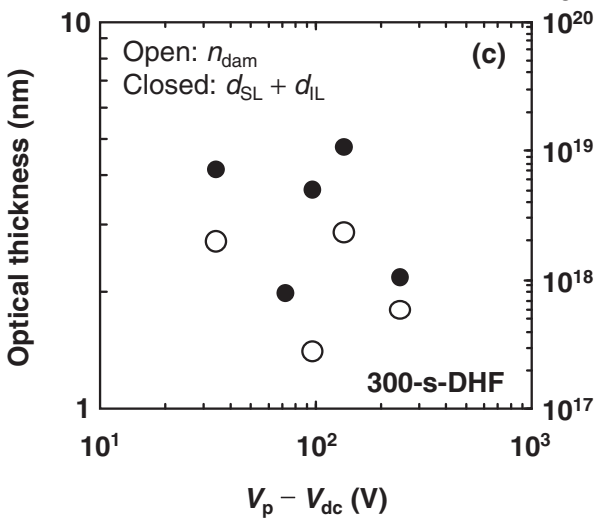

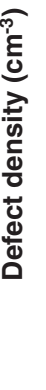

Figure 9. Dependences of $\left(d_{\mathrm{SL}}+d_{\mathrm{IL}}\right)$ (on the left axis) and $n_{\mathrm{dam}}$ (on the right axis) on the average incident ion energy in various DHF steps. (a) Before DHF treatment. (b) After 60-s-DHF. (c) After 300-s-DHF.

of incident ions. As seen in Fig. 8 and Fig. 9a, a larger $\bar{E}_{\text {ion }}$ result in thicker $d_{\mathrm{IL}}\left(d_{\mathrm{SL}}+d_{\mathrm{IL}}\right)$ and larger $n_{\mathrm{dam}}$. Compared to ICP, CCP has larger $\bar{E}_{\text {ion }}$ as deduced from Table I. However, $d_{\mathrm{IL}}$ by SE and $n_{\mathrm{dam}}$ by $1 / C^{2}-V$ technique for the CCP case are smaller than those for ICP even after $60-\mathrm{s}-\mathrm{DHF}$ wet etch. The mechanism explaining this counter-intuitive result is illustrated in Figs. 10. Figures 10 show comparisons of the profiles of $n_{\text {dam }}$ deduced from the energy (ICP: $-130 \mathrm{~V}$ and CCP: $-240 \mathrm{~V}$ ) and the flux of incident ions by the PPD range theory. In the range theory, the series of ion impacts is assumed to be the "cumulative" events, which is independent of the ion flux. (In Fig. 10, the value in the parenthesis is derived from the PPD range theory for the case of Ar plasma damage.) Compared to the ICP sample (Table I), the CCP sample has a wider $n_{\text {dam }}$ distribution due to the higher $\bar{E}_{\text {ion }}$ and straggling but lower in the $n_{\text {dam }}$ due to the lower ion flux. Moreover, one should consider UV radiation as a cause of damage creation mechanism. As listed in Table I, the ICP has the density higher than the CCP. This results in heavier damage for the case of the ICP. Although we do not have detailed optical emission spectra for the series of the present experiments, the ICP exposure created higher $n_{\text {dam }}$ as illustrated in Fig. 10. Note that the SE and $1 / C^{2}-V$ techniques have the detection limits as indicated in Figs. 10. The intersections with each detection limit define the measured values such as $d_{\mathrm{SL}}, d_{\mathrm{IL}}$, and, the areas as highlighted. Due to the lower defect density, $d_{\mathrm{CCP}}\left(=d_{\mathrm{SL}}+d_{\mathrm{IL}}\right)$ for CCP becomes thinner than $d_{\mathrm{ICP}}$ by

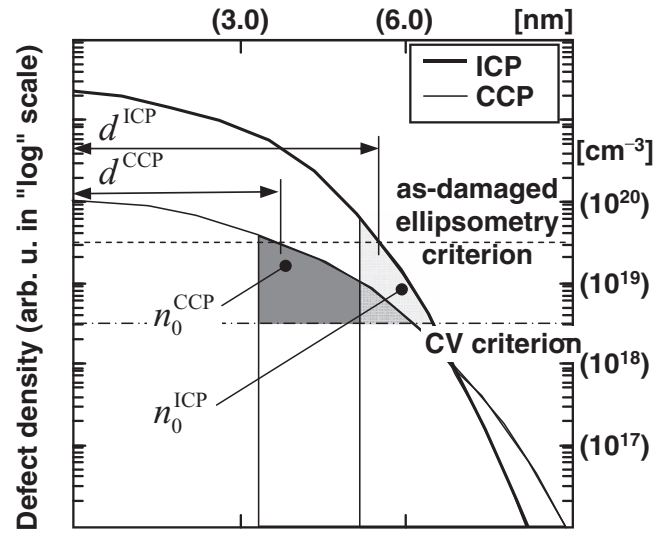

Depth (arb. u. in "linear" scale)

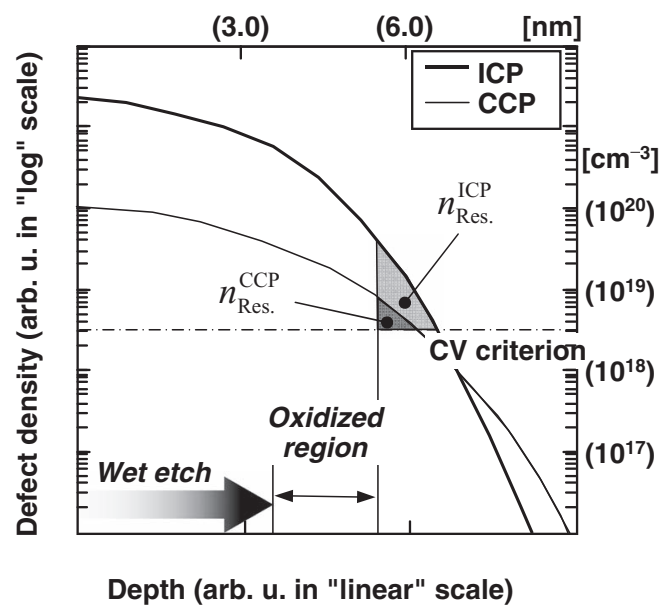

Figure 10. Schematic illustrations for the profiles of $n_{\mathrm{dam}}$ in the present ICPand CCP-damaged samples; (a) as-damaged and (b) wet-etched structures. Highlighted areas correspond to the $n_{\mathrm{dam}}$ identified by the $1 / C^{2}-V$ technique. The labels "as-damaged ellipsometry criterion" and "CV criterion" mean the detection limits by SE and $C-V$ method, respectively.

SE with the detection limit as indicated in Fig. 10. Moreover, $n_{0}^{\mathrm{CCP}}$ assigned by the $1 / C^{2}-V$ is smaller than $n_{0}^{\text {ICP }}$. A lower ion flux results in a lower $n_{\mathrm{dam}}$ in the deep substrate region-below the detection limit. Therefore, when comparing the PPD, one should pay careful attention to the fact that not only incident energy, but also the ion flux strongly affects the measured defect density. Figure 11 schematically illustrates how the defects distribute and the PPD values are measured. The present $V_{\mathrm{b}}$-sweeping range in the $C-V$ test corresponds to the depth of $3-5 \mathrm{~nm}$ in the substrate, deduced from the wafer resistivitylower resistive wafer is indispensible to address PPD distributed in a few nanometers. The changes of $n_{\mathrm{dam}}$ occur in the surface region within a few nanometers. It is concluded that the $1 / C^{2}-V$ technique is a powerful tool for PPD quantification, but one should pay careful attention to the profiles of defects in the nano-scale regime as well as the detection limits of analysis techniques employed.

Device performance degradation by residual PPD.- Finally, we investigate the effect of residual defects on MOSFET performance. The defects are considered to create additional energy levels in the bandgap ("band-gap states"), ${ }^{58,59}$ which plays the role of a carrier trap site. Thus, the defects induce drain current decrease ${ }^{15}$ due to the latent defects. With the present methodology, we can speculate that the residual density after DHF wet-etch widely employed in mass productions is determined around $5 \times 10^{18} \mathrm{~cm}^{-3}$. From TechnologyComputer Aided Design (TCAD) simulations, a residual defect 


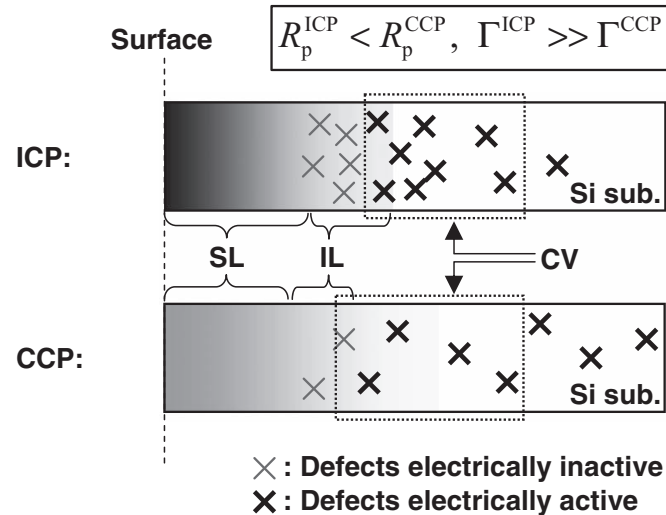

Figure 11. Schematic illustrations for the distributions of defects in the present ICP- and CCP-damaged samples. $\left(\left|V_{\mathrm{dc}}^{\mathrm{ICP}}\right|<\left|V_{\mathrm{dc}}^{\mathrm{CCP}}\right|\right)$ Owing to the higher incident ion energy in the CCP, the defects are distributed deeply in the $\mathrm{Si}$ substrate. However, the lower ion flux results in smaller $n_{\mathrm{dam}}$, in particular, in the deeper Si substrate region.

density of $\sim 5 \times 10^{18} \mathrm{~cm}^{-3}$ leads to $\sim 3 \%$ decrease of drain current for the 45 -nm-technology node. ${ }^{45}$ This amount is significant for LSI circuit designers. Thus, both a precise and quantitative identification of $n_{\mathrm{dam}}$ and an optimization of damaged layer removal process are necessary for future low damage process designs.

\section{Conclusions}

A quantitative nano-scale characterization of PPD was demonstrated for ICP and CCP sources using a $C-V$ technique combined with SE. A DHF wet-etch step was carried out to address the residual defect density in damaged samples, which is key to MOSFET performance design. The optical thickness by SE was compared with the electrical oxide thicknesses by the $C-V$ technique. The defect density $\left(n_{\mathrm{dam}}\right)$ assigned by the $C-V$ technique under various process treatments suggests that one should pay careful attention to the nature of PPD such as the profiles of defects as well as the sensitivity of each analysis techniques in the nano-scale regime. The present methodology should be implemented for designing low-damage process such as ALEt and addressing the residual defect impacts on the performance of ultimately-scaled devices.

\section{Acknowledgment}

This work was supported in part by a Grant-in-Aid for Scientific Research (B) 25630293 from the Japan Society for the Promotion of Science.

\section{References}

1. K. P. Cheung, Plasma Charging Damage (Springer, Heidelberg, 2001).

2. K. Eriguchi and K. Ono, J. Phys. D, 41, 024002 (2008).

3. A. Martin, J. Vac. Sci. \& Technol. B, 27, 426 (2009).

4. K. Eriguchi, Y. Uraoka, H. Nakagawa, T. Tamaki, M. Kubota, and N. Nomura, Jpn. J. Appl. Phys., 33, 83 (1994).

5. K. Eriguchi, Y. Nakakubo, A. Matsuda, M. Kamei, Y. Takao, and K. Ono, Jpn. J. Appl. Phys., 49, 04DA18 (2010)

6. T. Yunogami and T. Mizutani, J. Appl. Phys., 73, 8184 (1993).

7. M. Okigawa, Y. Ishikawa, and S. Samukawa, J. Vac. Sci. Technol. B, 21, 2448 (2003)

8. J. Lee and D. B. Graves, J. Phys. D, 44, 325203 (2011)

9. G. S. Oehrlein, Materials Sci. Eng. B, 4, 441 (1989).

10. K. Egashira, K. Eriguchi, and S. Hashimoto, IEDM Tech. Dig., 563 (1998).

11. S. A. Vitale and B. A. Smith, J. Vac. Sci. Technol. B, 21, 2205 (2003).

12. T. Ohchi, S. Kobayashi, M. Fukasawa, K. Kugimiya, T. Kinoshita, T. Takizawa, S. Hamaguchi, Y. Kamide, and T. Tatsumi, Jpn. J. Appl. Phys., 47, 5324 (2008)
13. K. Eriguchi, Y. Nakakubo, A. Matsuda, M. Kamei, Y. Takao, and K. Ono, Jpn. J. Appl. Phys., 49, 08JC02 (2010).

14. Y. Nakakubo, A. Matsuda, M. Fukasawa, Y. Takao, T. Tatsumi, K. Eriguchi, and K. Ono, Jpn. J. Appl. Phys., 49, 08JD02 (2010).

15. K. Eriguchi, Y. Nakakubo, A. Matsuda, Y. Takao, and K. Ono, IEEE Electron Device Lett., 30, 1275 (2009).

16. K. Eriguchi, A. Matsuda, Y. Nakakubo, M. Kamei, H. Ohta, and K. Ono, IEEE Electron Device Lett., 30, 712 (2009).

17. K. Eriguchi, M. Kamei, Y. Takao, and K. Ono, Proc. International Conference on Integrated Circuit Design \& Technology, 94 (2010).

18. K. Eriguchi, Y. Nakakubo, A. Matsuda, Y. Takao, and K. Ono, Jpn. J. Appl. Phys., 49, 056203 (2010)

19. S. M. Sze, Semiconductor Devices, Physics and Technology, 2nd ed. (John Wiley \& Sons, Inc., Hoboken, NJ, 2002)

20. S. M. Sze, VLSI Technology, 2nd ed. (McGraw-Hill Book Company, New York, 1988).

21. K. J. Kanarik, T. Lill, E. A. Hudson, S. Sriraman, S. Tan, J. Marks, V. Vahedi, and R. A. Gottscho, J. Vac. Sci. \& Technol. A, 33, 020802 (2015).

22. G. S. Oehrlein, R. M. Tromp, J. C. Tsang, Y. H. Lee, and E. J. Petrillo, J. Electrochemical Society, 132, 1441 (1985).

23. S. C. Vitkavage and E. A. Irene, J. Appl. Phys., 64, 1983 (1988)

24. A. Matsuda, Y. Nakakubo, Y. Takao, K. Eriguchi, and K. Ono, Thin Solid Films, 518 , 3481 (2010).

25. P. K. Giri, S. Tripurasundari, G. Raghavan, B. K. Panigrahi, P. Magudapathy, K. G. M. Nair, and A. K. Tyagi, J. Appl. Phys., 90, 659 (2001).

26. Y. Nakamura, T. Tatsumi, S. Kobayashi, K. Kugimiya, T. Harano, A. Ando, T. Kawase, S. Hamaguchi, and S. Iseda, J. Vac. Sci. \& Technol. A, 25, 1062 (2007).

27. M. Fukasawa, Y. Nakakubo, A. Matsuda, Y. Takao, K. Eriguchi, K. Ono, M. Minami, F. Uesawa, and T. Tatsumi, J. Vac. Sci. Technol. A, 29, 041301 (2011).

28. I. W. Wu, R. A. Street, and J. J. C. Mikkelsen, J. Appl. Phys., 63, 1628 (1988).

29. K. T. Sung, S. W. Pang, M. W. Cole, and N. Pearce, J. Electrochemical Society, 142, 206 (1995).

30. O. O. Awadelkarim, P. I. Mikulan, T. Gu, K. A. Reinhardt, and Y. D. Chan, J. Appl. Phys., 76, 2270 (1994)

31. M. Koyama, C.-w. Cheong, K. Yokoyama, and I. Ohdomari, Jpn. J. Appl. Phys., 11, 6682 (1997).

32. O. O. Awadelkarim, T. Gu, R. A. Ditizio, P. I. Mikulan, S. J. Fonash, J. F. Rembetski, and Y. D. Chan, Electron Device Letters, IEEE, 14, 167 (1993).

33. I. W. H. Connick, A. Bhattacharyya, K. N. Ritz, and W. L. Smith, J. Appl. Phys., 64, 2059 (1988)

34. K. Eriguchi, Y. Nakakubo, A. Matsuda, M. Kamei, H. Ohta, H. Nakagawa, S. Hayashi, S. Noda, K. Ishikawa, M. Yoshimaru, and K. Ono, IEDM Tech. Dig., 443 (2008)

35. G. G. George and E. M. Gunnersen, 7th International Conference on the Physics of Semiconductors, 385 (1964).

36. A. S. Yapsir, P. Hadizad, T. M. Lu, J. C. Corelli, J. W. Corbett, W. A. Lanford, and H. Bakhru, Phys. Rev. B, 37, 8982 (1988).

37. S. M. Sze and K. K. Ng, Physics of Semiconductor Devices, 3rd ed. (WileyInterscience, Hoboken, NJ, 2007)

38. K. Eriguchi, A. Matsuda, Y. Takao, and K. Ono, Jpn. J. Appl. Phys., 53, 03 DE02 (2014).

39. J. Lindhard, M. Scharff, and H. E. Schiott, Mat. Fys. Medd. K. Dan. Vidensk. Selsk., 33, 1 (1963).

40. J. F. Gibbons, Proceedings of the IEEE, 60, 1062 (1972).

41. M. Posselt, B. Schmidt, C. S. Murthy, T. Feudel, and K. Suzuki, J. Electrochem. Soc., 144, 1495 (1997).

42. J. D. Plummer, M. Deal, and P. B. Griffin, Silicon VLSI Technology, Fundamentals, Practice and Modeling (Prentice Hall, New Jersey, 2000).

43. J. F. Gibbons, Proceedings of the IEEE, 56, 295 (1968).

44. K. Eriguchi, Y. Nakakubo, A. Matsuda, M. Kamei, Y. Takao, and K. Ono, Jpn. J. Appl. Phys., 50, 08KD04 (2011).

45. SIA, The International Technology Roadmap for Semiconductors, 2012 update (2012).

46. K. Eriguchi, A. Ohno, D. Hamada, M. Kamei, and K. Ono, Thin Solid Films, 516, 6604 (2008).

47. K. Eriguchi, A. Ohno, D. Hamada, M. Kamei, H. Fukumoto, and K. Ono, Jpn. J. Appl. Phys., 47, 2446 (2008)

48. A. Matsuda, Y. Nakakubo, M. Kamei, Y. Takao, K. Eriguchi, and K. Ono, Ext. Abs. Solid State Dev. Mat., 346 (2009).

49. H. Ohta and S. Hamaguchi, J. Vac. Sci. \& Technol., A19, 2373 (2001).

50. H. Ohta, T. Nagaoka, K. Eriguchi, and K. Ono, Jpn. J. Appl. Phys., 48, 020225 (2009)

51. W. D. Wilson, L. G. Haggmark, and J. P. Biersack, Phys. Rev. B, 15, 2458 (1977).

52. I. P. Batra, F. F. Abraham, and S. Ciraci, Phys. Rev. B, 35, 9552 (1987).

53. W. K. Leung, R. J. Needs, G. Rajagopal, S. Itoh, and S. Ihara, Phys. Rev. Lett., 83, 2351 (1999).

54. L. Colombo, Annual Review of Materials Research, 32, 271 (2002)

55. M. Tang, L. Colombo, J. Zhu, and T. Diaz de la Rubia, Phys. Rev. B, 55, 14279 (1997).

56. D. B. Graves and D. Humbird, Appl. Surf. Sci., 192, 72 (2002).

57. H. Wada, M. Agata, K. Eriguchi, A. Fujimoto, T. Kanashima, and M. Okuyama, $J$. Appl. Phys., 88, 2336 (2000).

58. S. K. Estreicher, J. L. Hastings, and P. A. Fedders, Appl. Phys. Lett., 70, 432 (1997).

59. P. A. Schultz, Phys. Rev. Lett., 96, 246401 (2006). 